\title{
Numerical simulation of three-dimensional breaking waves and its interaction with a vertical circular cylinder
}

\author{
Zhihua Xie ${ }^{1,2,3,4 *}$, Lin Lu², Thorsten Stoesser ${ }^{1}$, Jianguo Lin ${ }^{5}$, Dimitrios Pavlidis ${ }^{3}$, Pablo Salinas ${ }^{3}$, \\ Christopher C. Pain ${ }^{3}$, Omar K. Matar ${ }^{4}$
}

${ }^{1}$ School of Engineering, Cardiff University, Cardiff, CF24 3AA, UK

${ }^{2}$ State Key Laboratory of Coastal and Offshore Engineering, Dalian University of Technology, Dalian,116024, China.

${ }^{3}$ Department of Earth Science and Engineering, Imperial College London, London, SW7 2AZ, UK

${ }^{4}$ Department of Chemical Engineering, Imperial College London, London, SW7 2AZ, UK

${ }^{5}$ College of Environmental Science and Engineering, Dalian Maritime University, Dalian,116026, China.

*E-mail: zxie@ cardiff.ac.uk

\begin{abstract}
Wave breaking plays an important role in wave-structure interaction. A novel control volume finite element method with adaptive unstructured meshes is employed here to study three-dimensional breaking waves. The numerical framework consists of a 'volume of fluid' type method for the interface capturing and adaptive unstructured meshes to improve computational efficiency. The numerical model is validated against experimental measurements of breaking wave over a sloping beach and is then used to study the breaking wave impact on a vertical circular cylinder on a slope. Detailed complex interfacial structures during wave impact, such as plunging jet formation and splash-up are captured in the simulation, demonstrating the capability of the present method.
\end{abstract}

KEY WORDS: Breaking waves; volume of fluid method; three-dimensional simulation; Navier-Stokes equation; adaptive unstructured mesh.

\section{INTRODUCTION}

Wave breaking plays an important role in marine hydrodynamics, wave-structure interaction, air-sea interaction, surf zone dynamics, and nearshore sediment transport. Several comprehensive reviews of breaking waves and wave mechanics can be found in [1-3].

Wave-structure interaction is a key aspect in the safe and cost-effective design of coastal and offshore structures, and marine renewable devices. In order to roughly predict the hydrodynamic loads on structures, the Morison equation and potential flow theory have been widely used in the literatures. However, it is challenge to consider breaking wave impact on the structures by using these two approaches, especially when there are splash-up and air entrainment ${ }^{[4]}$.

With developments of CFD (computational fluid dynamics) and increases in computer power, recent models for studying free surface flows, including breaking waves, solve the Navier-Stokes equations coupled with a free surface calculation (see $\mathrm{Lin}^{[5]}$ for comprehensive modelling applications and methodologies for water waves and McSherry et $\mathrm{a}^{[6]}$ for large-eddy simulation of free surface flows). There are several numerical studies on breaking waves in the literature based on one-phase flow model in two-dimension (2D) ${ }^{[7-10]}$ and three-dimension (3D) ${ }^{[11,12]}$, 
in which only the flow in the water is considered in the computations. The pressure in the air is taken as a constant, and the boundary conditions are approximately specified at the free surface. In order to take the effect of air phase into account for wave breaking, several 2D two-phase flow model, in which both flows in the air and water are solved, have been developed to study the details of breaking waves in the surf zone ${ }^{[13,14]}$, overturning jet during wave breaking ${ }^{[15]}$ and the effect of wind on breaking waves ${ }^{[16,17]}$. Several 3D two-phase flow models have also been developed to understand 3D breaking waves in a periodic space domain ${ }^{[18]}$, over a plane beach ${ }^{[19]}$ and over a complex topography ${ }^{[20]}$, which have provided much insight into the kinematics and dynamics of breaking waves, including the overturning jet and the subsequent splash-up process. Previous investigations have greatly improved our knowledge of breaking waves, however, little attention has been given for the numerical study on the 3D wave action on a vertical circular cylinder over a slope.

In this study, a 3D two-phase flow model with adaptive unstructured meshes is developed to investigate 3D breaking wave interaction with a vertical circular cylinder along a constant sloping beach, which can provide detailed information on the impact force during wave breaking. The usage of adaptive unstructured meshes is helpful to reduce the computational efforts in CFD simulations and is also easy to deal with the problems with irregular boundaries. The description of the mathematical model for the two-phase flow is described in next section. The numerical method is presented after that. Both 2D overturning waves and 3D breaking wave impacting on a cylinder are simulated in the results and discussion section. Detailed computational results of the water surface profiles associated with the adaptive unstructured meshes are shown and discussed. Finally, conclusions are drawn.

\section{MATHMATICAL MODEL}

A multi-fluid modelling framework has been developed based on the multi-component modelling approach with information on interfaces embedded into the continuity equations. In two-phase flows, let $\alpha_{i}$ be the mass fraction of phase $i$, where $i=1,2$, the density and dynamic viscosity of phase $i$ are $\rho_{i}$ and $\mu_{i}$, respectively. A constraint on the system is:

$$
\sum_{i=1}^{2} \alpha_{i}=1
$$

For each fluid component $i$, the conservation of mass may be denoted as:

$$
\frac{\partial}{\partial t}\left(\alpha_{i}\right)+\nabla \cdot\left(\alpha_{i} \boldsymbol{u}\right)=0, \quad i=1,2
$$

and the equations of motion of an incompressible fluid can be written as:

$$
\frac{\partial(\rho \boldsymbol{u})}{\partial t}+\nabla \cdot(\rho \boldsymbol{u} \otimes \boldsymbol{u})=-\nabla p+\nabla \cdot\left[\mu\left(\nabla \boldsymbol{u}+\nabla^{\mathrm{T}} \boldsymbol{u}\right)\right]+\rho \boldsymbol{g}+\sigma \kappa \boldsymbol{n} \delta
$$

where where $\mathrm{t}$ is the time, $\mathrm{u}$ is velocity vector, $\mathrm{p}$ is the pressure, the bulk density is $\rho=\rho_{1} \alpha_{1}+\rho_{2} \alpha_{2}$, the bulk dynamic viscosity is $\mu=\mu_{1} \alpha_{1}+\mu_{2} \alpha_{2}, g$ is the gravitational acceleration vector, $\sigma$ is the surface tension coefficient, $\kappa=\nabla \cdot \boldsymbol{n}$ is the interfacial curvature, $\boldsymbol{n}$ is the interface unit normal, and $\delta$ is the Dirac delta function.

\section{NUMERICAL METHOD}

In the present study, a transient, mixed, control-volume and finite-element formulation is used to discretise the governing equations (Eq. 2 and Eq. 3). A finite volume discretisation of the continuity equations and a linear discontinuous Galerkin (DG) ${ }^{[21]}$ discretisation of the momentum equations are employed with backward Euler 
time stepping. Within each time-step, the equations are iterated upon using a projection-based pressure determination method until all equations are simultaneously balanced. The main numerical framework includes a finite element type P1DG-P2 (linear discontinuous velocity between elements and quadratic continuous pressure between elements) for multi-fluid flow problems, which ensures exact balance between buoyancy force and pressure gradient. The framework also features a novel interface capturing scheme based on compressive control volume advection method ${ }^{[21]}$, involving a high-order accurate finite element method to obtain fluxes on the control volume boundaries, where these fluxes are subject to flux-limiting using a normalised variable diagram approach to obtain bounded and compressive solutions for the interface. The implementation of capillary/surface tension force in the framework using an unstructured mesh minimises spurious velocities often found in interfacial flows $^{[22]}$. Finally, use of anisotropic unstructured mesh adaptivity ${ }^{[23]}$ allows the grid resolution to be concentrated in relatively important regions, such as the vicinity of interfaces, while lower resolution can be used in other regions; this leads to a significant gain in computational efficiency without sacrificing accuracy.

The numerical framework has been validated and employed to study various multiphase flow problems ${ }^{[24-27]}$. The detailed modelling of three-dimensional bubbles, droplet and liquid films can be found in Xie et al ${ }^{[22,28]}$.

\section{RESULTS AND DISCUSSION}

\section{D Breaking Wave Over a Sloping Beach}

In this section, we simulate a two-dimensional overturning solitary wave and compare quantitatively with the experiment $^{[29]}$ for a breaking solitary wave splash-up on a 1:15 sloping beach. We followed the same computational setup in our previous studies with a two-phase flow solver based on the finite volume method on a fixed grid ${ }^{[15,20]}$. At the inlet, the solitary wave is generated by giving the water surface profile and the water particle velocities ${ }^{[15]}$, whereas the no-slip wall boundary condition is applied at the sloping beach and zerogradient boundary conditions are applied at the top and outlet of the computational domain.

Fig. 1 shows the evolution of zoom in region for the solitary wave before and after wave breaking. It can be seen that the development of plunging jet, jet impingement and generation of splash-up are reproduced in the simulation. Sharp air-water interface is captured by the adaptive unstructured mesh, with fine mesh in the vicinity of the interface and coarse mesh away from the interface. A detailed comparison of the plunging jet, at the time of wave overturning, between the simulation and experimental results is shown in Fig. 2. Good agreement is obtained in the present study in terms of wave shape and breaking location, demonstrating the capability of the present method in simulation wave breaking problems.
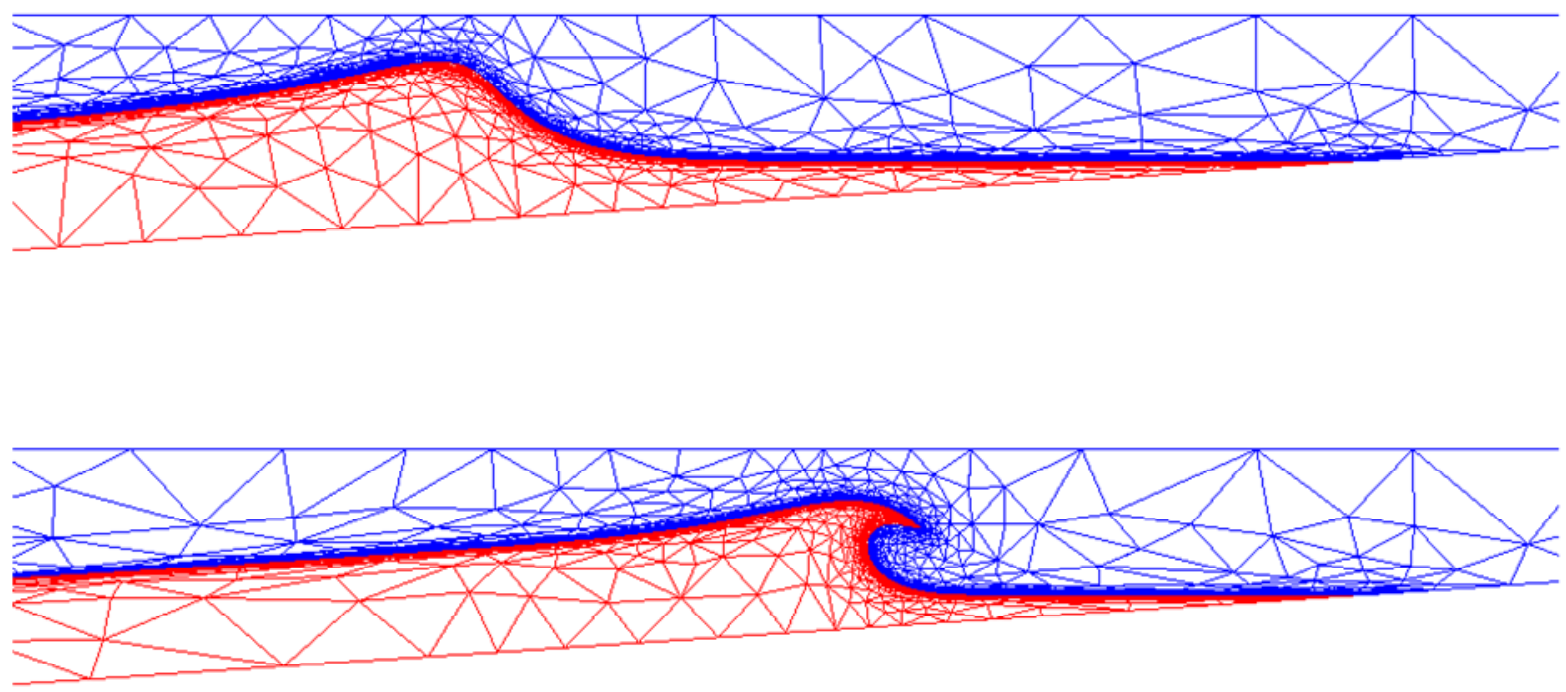


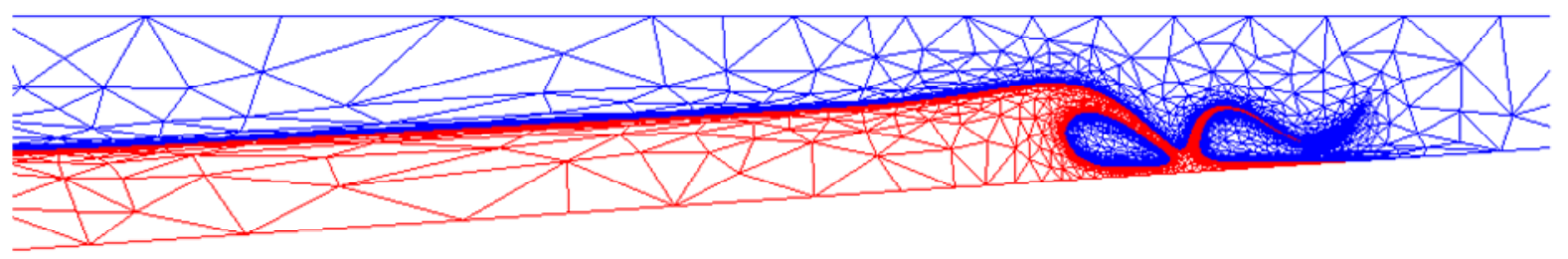

Fig. 1 Evolution of a solitary breaking wave with $H / D=0.45$ along a 1: 15 sloping beach. Water and air and their associated fully unstructured mesh are shown in red and blue colors.

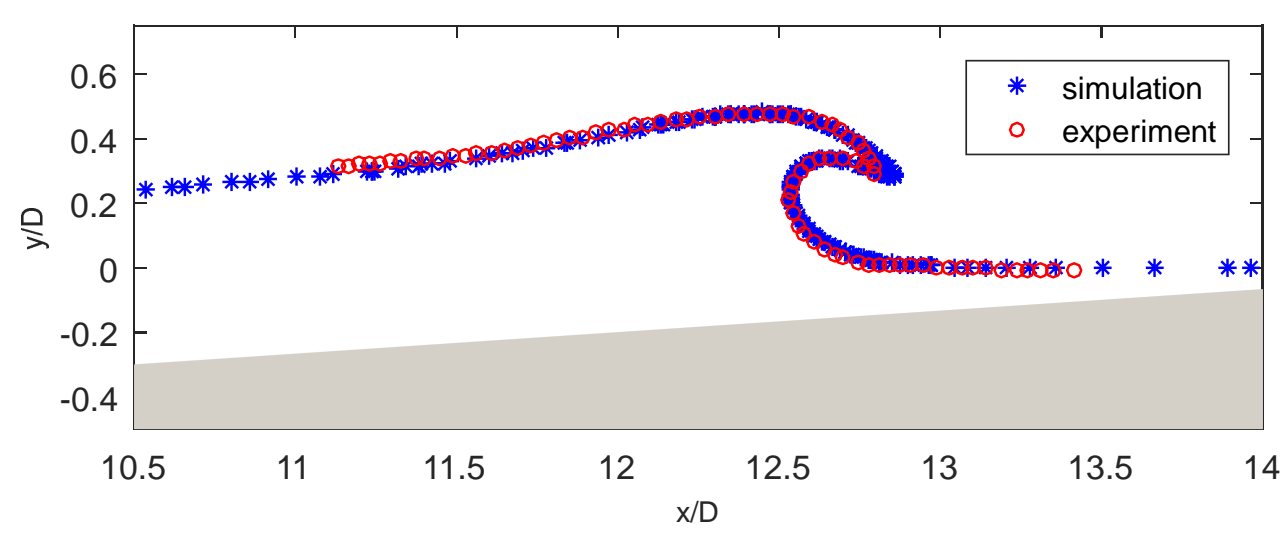

Fig. 2 Comparison between simulation and experimental measurements ${ }^{[29]}$ for the wave surface profile during wave overturning on a sloping beach.

\section{D Breaking Wave Impact on a Vertical Circular Cylinder}

A three-dimensional solitary breaking wave over a constant slope is investigated here. In the computation, the origin of the coordinates is located at the still water level in the centre of the toe of the slope and all lengths are normalized by the water depth $D=0.3048 \mathrm{~m}$. The computational setup is similar to the case for a 3D breaking wave without a cylinder ${ }^{[20]}$, in which the slope is $1: 15$ and the incident plane solitary wave with the ratio of wave height to water depth is $H / D=0.45$. A vertical circular cylinder with a diameter of $d / D=0.33$ is considered here and it is located at a distance of $x=12.5 D$ from the toe of the slope. The computational domain, which has a length of $19.75 D$, width of $1.3 D$, and height of $1.75 D$, is discretized by an adaptive fullyunstructured mesh, with minimum meshes of $\Delta x_{\min } / D=0.02, \Delta y_{\min } / D=0.02$ and $\Delta z_{\min } / D=0.05$ in the streamwise, vertical and spanwise directions, respectively.

Fig. 3 shows snapshots of detailed views of the solitary wave before, during and after wave breaking. Before wave breaking, it can be seen that the wave crest becomes steep due to the shoaling effect (Fig. 3(a)). A typical threedimensional overturning jet just ahead of the cylinder can be seen in Fig. 3(b) during wave breaking. The breaking wave impacts with the vertical cylinder and 3D complex plunging jet is developed in Fig. 3(c). The adaptive fullyunstructured meshes are also shown in Fig. 3 and it can be seen that fine mesh is used in the vicinity of the interface and the region near the cylinder whereas coarse mesh is used away from the interface. This has the advantage of reducing computational effort without sacrificing accuracy. 

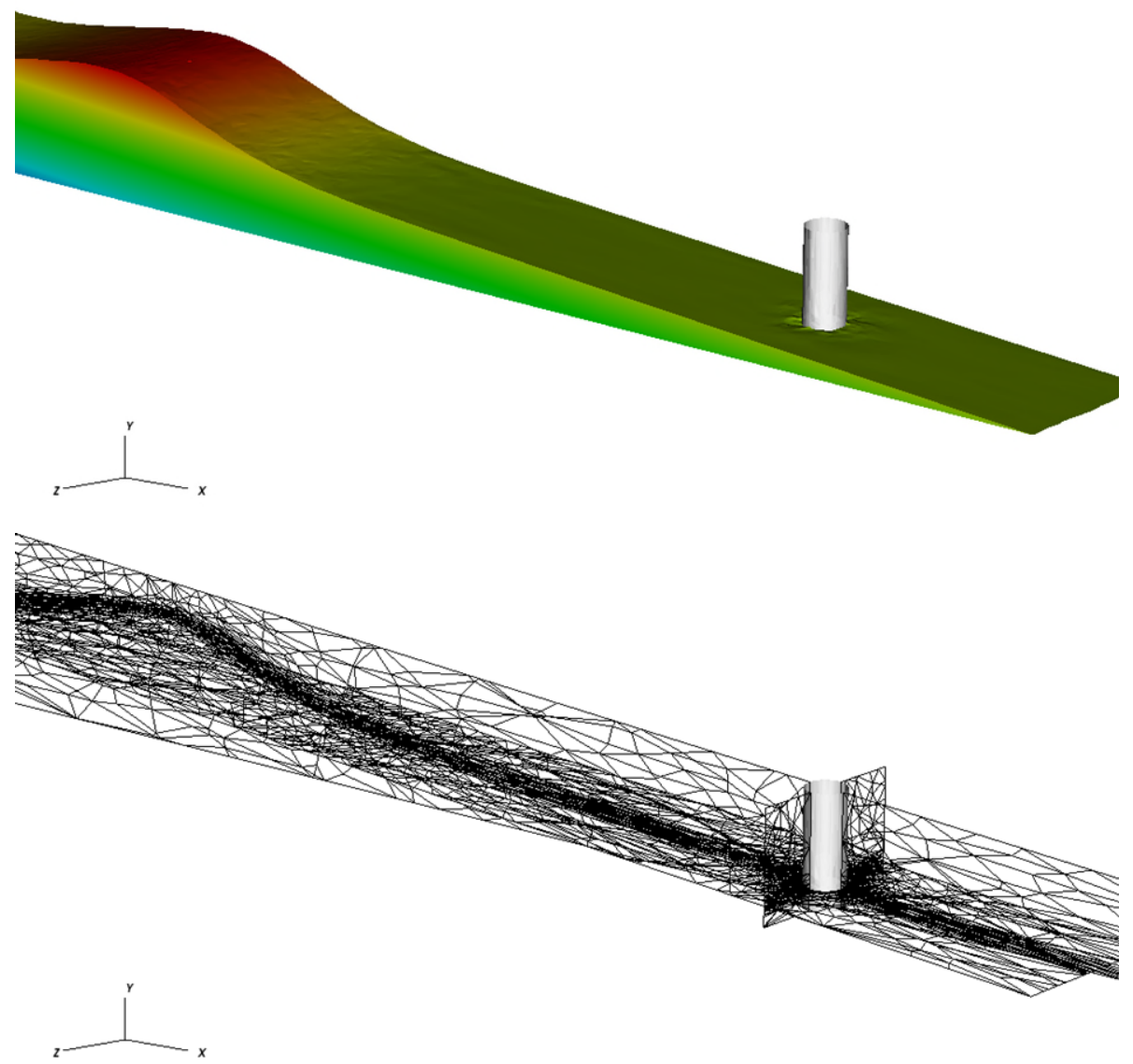

(a) before wave breaking

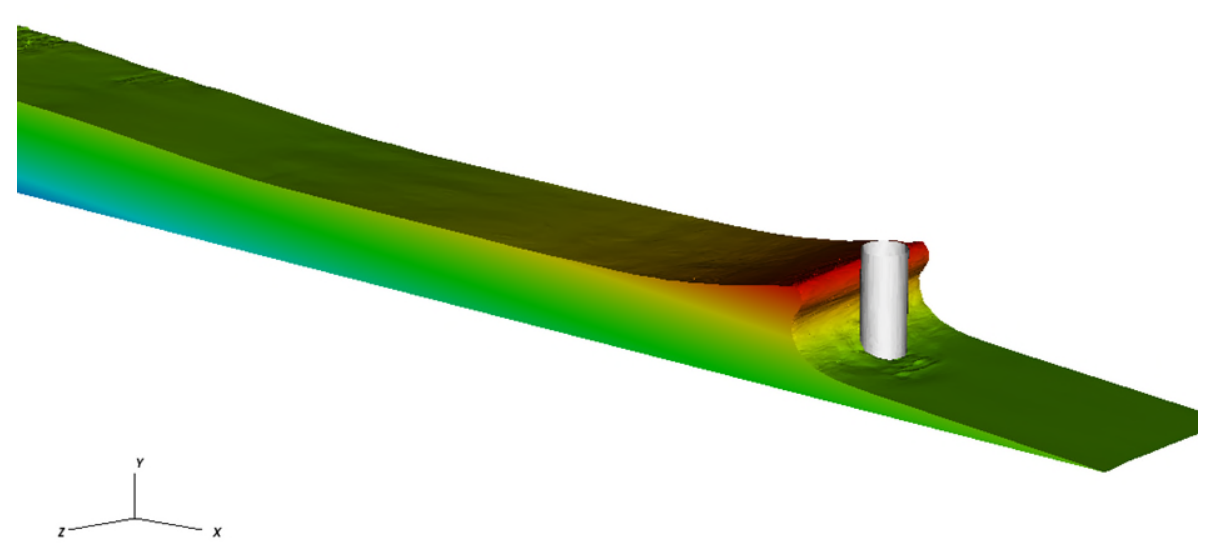




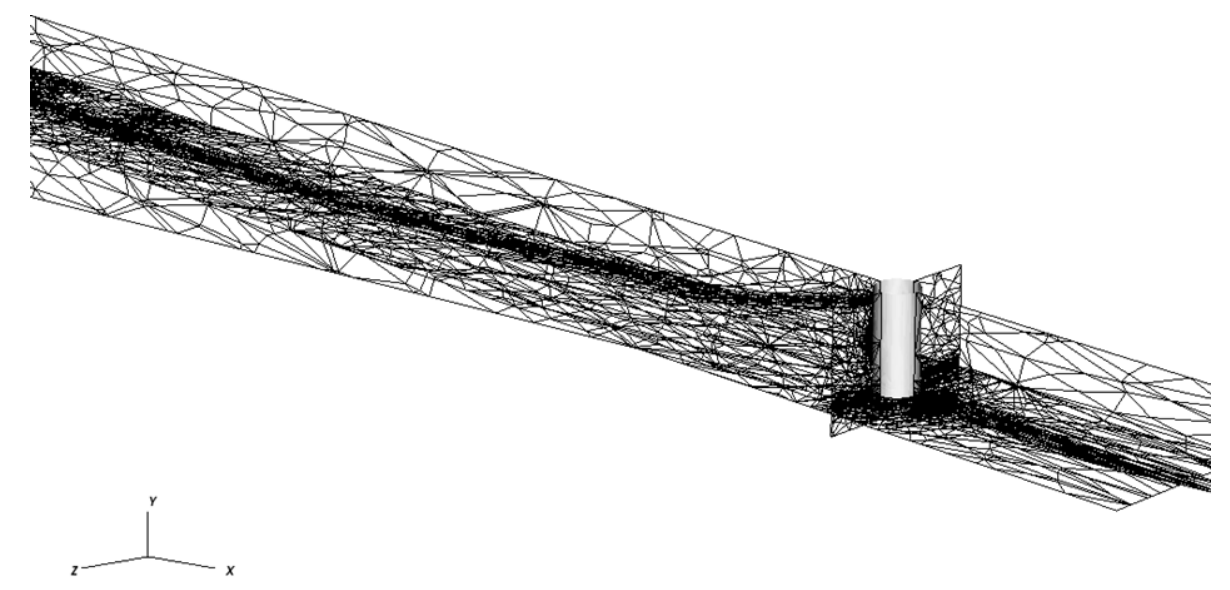

(b) during wave breaking

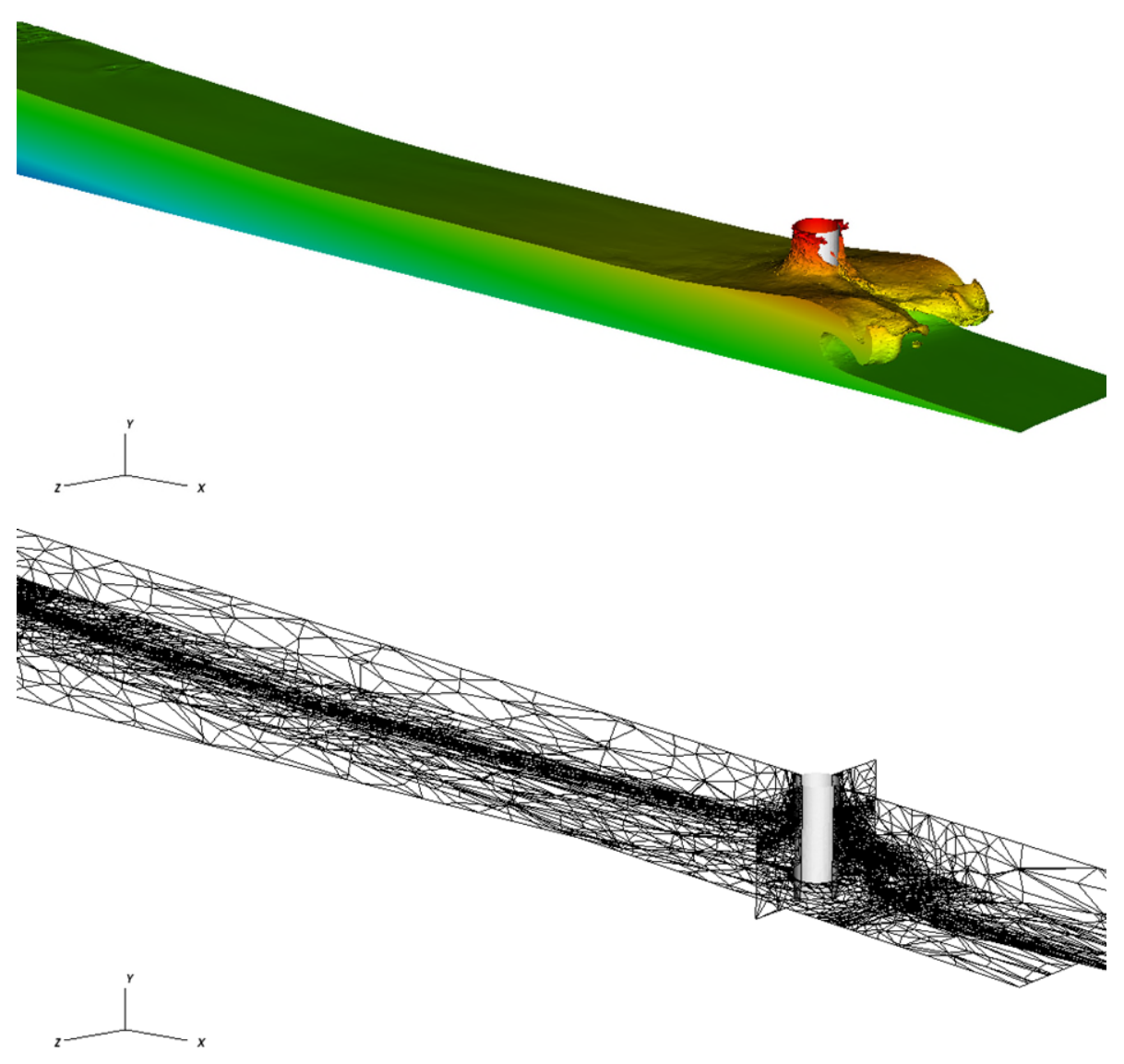

(c) after wave breaking

Fig. 3 Detailed views of the water wave surface before (a), during (b) and after (c) wave breaking. Top pannel shows the water surfaces colored based on local values of $y / D$ and bottom pannel shows the fully-unstructured mesh corresponded to the top pannel along three slices.

Fig. 4 shows the streamwise-component impact force on the vertical cylinder during wave propagation along the sloping beach. It can be seen that the force is very small when the wave is far away from the cylinder. It gradually increases and obtains the maximum value when the plunging jet hits the cylinder. Then impact force gradually decreases when the breaking wave passes by and there is some oscillation due to three-dimensional effect of the breaking waves. 


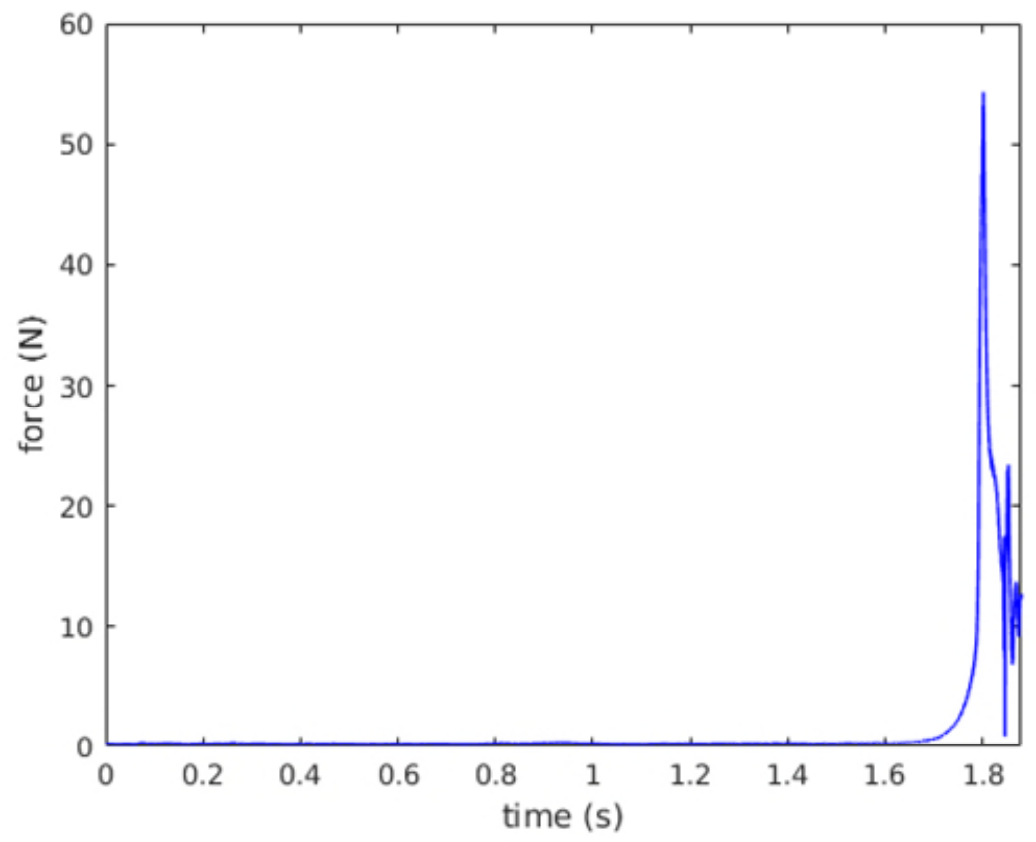

Fig. 4 Streamwise impact force on the vertical circular cylinder for the breaking wave along the sloping beach.

\section{CONCLUSIONS}

In this paper, a control volume finite element method with adaptive anisotropic unstructured meshes has been used for three-dimensional breaking waves, which can modify and adapt unstructured meshes to better represent the underlying physics of interfacial problems and reduce computational effort without sacrificing accuracy.

The numerical framework has been validated for a 2D breaking wave over a sloping beach, in which a good agreement of the water surface profile between the simulation and experimental measurements is obtained. The present framework has been further employed to investigate a 3D breaking wave and its impact on a vertical cylinder along a slope. Development of plunging jet formation and complex interfacial structures during wave impact have been captured by the present method. The results presented here established with sufficient confidence that this method can be used to successfully model breaking waves and wave-structure interaction in a wide range of applications.

\section{ACKNOWLEDGEMENTS}

The first and second authors would like to acknowledge the financial support by the National Natural Science Foundation of China with grant NO. 51490673 and Open Awards of the State Key Laboratory of Coastal and Offshore Engineering. This work was also funded by the EPSRC MEMPHIS multiphase Programme Grant (number EP/K003976/1) and funding from the European Union Seventh Framework Programme (FP7/20072013) under grant agreement NO. 603663 for the research project PEARL (Preparing for Extreme And Rare events in coastaL regions).

\section{REFERENCES}

[1] Peregrine D. H. Breaking waves on beaches [J]. Annual Review of Fluid Mechanics, 1983, 15: 149-178.

[2] Banner M. L., Peregrine D. H. Wave breaking in deep-water [J]. Annual Review of Fluid Mechanics, 1993, 25: 373-397.

[3] Mei C. C., Liu P. L. F. Surface-waves and coastal dynamics [J]. Annual Review of Fluid Mechanics, 1993, 25: 
[4] Kiger K. T., Duncan J. H. Air-entrainment mechanisms in plunging jets and breaking waves [J]. Annual Review of Fluid Mechanics, 2012, 44: 563-596.

[5] Lin P. Numerical Modeling of Water Waves: An introduction to engineers and scientists [M]. Taylor \& Francis, 2008.

[6] McSherry R. J., Chua K. V., Stoesser T. Large eddy simulation of free-surface flows [J]. Journal of Hydrodynamics, 2017, 29(1): 1-12.

[7] Lin P., Liu P. L. F. A numerical study of breaking waves in the surf zone [J]. Journal of Fluid Mechanics, 1998, 359: 239-264.

[8] Bradford S. F. Numerical simulation of surf zone dynamics [J]. Journal of Waterway, Port, Coastal, and Ocean Engineering-ASCE, 2000, 126(1): 1-13.

[9] Zhao Q, Armfield S., Tanimoto K. Numerical simulation of breaking waves by a multi-scale turbulence Model

[J]. Coastal Engineering, 2004, 51(1): 53-80.

[10] Shao S. Simulation of breaking wave by SPH method coupled with k-E model [J]. Journal of Hydraulic Research, 2006, 44(3): 338-349.

[11] Christensen E. D., Deigaard R. Large eddy simulation of breaking waves [J]. Coastal Engineering, 2001, 42(1): 53-86.

[12] Watanabe Y., Saeki H., Hosking R. J. Three-dimensional vortex structures under breaking waves [J]. Journal of Fluid Mechanics, 2005, 545: 291-328.

[13] Wang Z., Zou Q., Reeve D. E. Simulation of spilling breaking waves using a two phase flow CFD model [J]. Computers \& Fluids, 2009, 38(10): 1995-2005.

[14] Xie Z. Two-phase flow modelling of spilling and plunging breaking waves [J]. Applied Mathematical Modelling, 2013, 37(6): 3698-3713.

[15] Xie Z. Numerical study of breaking waves by a two-phase flow model. International Journal for Numerical Methods in Fluids, 2012, 70(2): 246-268.

[16] Xie Z. Numerical modelling of wind effects on breaking solitary waves [J]. European Journal of MechanicsB/Fluids, 2014, 43: 135-147.

[17] Xie Z. Numerical modelling of wind effects on breaking waves in the surf zone [J]. Ocean Dynamics, in press, 2017. doi: 10.1007/s10236-017-1086-8

[18] Lubin P., Vincent S., Abadie S., Caltagirone J. P. Three-dimensional large eddy simulation of air entrainment under plunging breaking waves [J]. Coastal Engineering, 2006, 53(8): 631-655.

[19] Lakehal D., Liovic P. Turbulence structure and interaction with steep breaking waves [J]. Journal of Fluid Mechanics, 2011, 674: 522-577.

[20] Xie Z. A two-phase flow model for three-dimensional breaking waves over complex topography [J]. Proceedings of the Royal Society A: Mathematical, Physical \& Engineering Sciences, 2015, 471: 20150101.

[21] Pavlidis D., Gomes J. L. M. A., Xie Z., Percival J. R., Pain C. C., Matar O. K. Compressive advection and multi-component methods for interface-capturing [J]. International Journal of Numerical Methods in Fluids, 2016, 80: 256-282. 
[22] Xie Z., Pavlidis D., Salinas P., Percival J. R., Pain C. C., Matar O. K. A balanced-force control volume finite element method for interfacial flows with surface tension using adaptive anisotropic unstructured meshes [J]. Computers and Fluids, 2016, 138: 38-50.

[23] Pain C. C., Umpleby A. P., de Oliveira C. R. E., Goddard A. J. H. Tetrahedral mesh optimisation and adaptivity for steady-state and transient finite element calculations [J]. Computer Methods in Applied Mechanics and Engineering, 2001, 190: 3771-3796.

[24] Xie Z., Pavlidis D., Percival J. R., Gomes J. L. M. A., Pain C. C., and Matar O. K. Adaptive unstructured mesh modelling of multiphase flows [J]. International Journal of Multiphase Flow, 2014, 67:104-110.

[25] Pavlidis D., Xie Z., Percival J. R., Gomes J. L. M. A., Pain C. C., and Matar O.K. Two- and three-phase horizontal slug flow simulations using an interface-capturing compositional approach [J]. International Journal of Multiphase Flow, 2014, 67: 85-91.

[26] Nowak E., Xie Z., Kovalchuk N. M., Matar O. K., and Simmons M. J. H. Bulk advection and interfacial flows in the binary coalescence of surfactant-laden and surfactant-free drops. Soft Matter, 2017, 13: 4616-4628.

[27] Adebayo I., Xie Z., Che Z., and Matar O. K. Doubly-excited pulse waves on thin liquid films flowing down an inclined plane: An experimental and numerical study [J]. Physical Review E, 2017, 96: 013118.

[28] Xie Z., Hewitt G.F., Pavlidis D., Salinas P., Pain C. C., Matar O. K. Numerical study of three-dimensional droplet impact on a flowing liquid film in annular two-phase flow [J]. Chemical Engineering Science, 2017, 166: 303-312.

[29] Li Y. Tsunamis: Non-breaking and breaking solitary wave run-up [C]. Ph.D. Thesis, California Institute of Technology, 2000. 